\title{
Contributing Ad Hoc Reviewers 2017
}

Rehabilitation Psychology publishes articles that capture the breadth of science and practice in rehabilitation psychology. To achieve this mission we rely on a diverse group of individuals who serve as ad hoc reviewers. We are grateful to the following individuals for their generous commitment of time and effort for the 2017 volume of Rehabilitation Psychology. If you would like to join this group of individuals by serving as an ad hoc reviewer please contact the editor at swegener@jhmi.edu.

\begin{tabular}{|c|c|}
\hline Amy Allen & Henk Eilander \\
\hline Herb Ames & Valerie Ellois* \\
\hline \multicolumn{2}{|l|}{ Tina Anctil } \\
\hline Derek Anderson & Ruth Farber \\
\hline Michael Anestis & Ana Faria \\
\hline Patricia Arenth & David Feldman \\
\hline Anne Arewasikporn & Liora Findler \\
\hline Trey Armstrong & $\begin{array}{l}\text { Pamela Fitzpatrick } \\
\text { Becca Floyd }\end{array}$ \\
\hline Marc Baertschi & Kitty-Rose Foley \\
\hline Martha Banks & Anjali Forber-Pratt \\
\hline Julie Baran* & Robert Fraser \\
\hline Sandra Bassett & Katherine Frey \\
\hline \multicolumn{2}{|l|}{ James Benton } \\
\hline Lisa Betthauser & Nicolette Gabel $^{*}$ \\
\hline Patrick Brice & Christina Garrison-Diehn* \\
\hline Dana Brickham & Maryam Gholami \\
\hline Roseina Britton* & Jenna Goesling \\
\hline Jo Brockway & Chantea Goetz \\
\hline Mary Brownsberger & Lance Goetz \\
\hline \multirow[t]{2}{*}{ Maggi Budd } & Yelena Goldin \\
\hline & Fernando Gonzalez \\
\hline Yue Cao & Christopher Graham \\
\hline Blathin Casey & Elizabeth Gromisch \\
\hline \multicolumn{2}{|l|}{ Denise Catalano } \\
\hline Elena Cavallini & Juliet Haarbauer-Krupa \\
\hline Jacob Yuichung Chan & Kimberley Haines \\
\hline Naomi Chaytor & Nicholas Harland \\
\hline Yuying Chen & H'Sien Hayward \\
\hline Amanda Childs* & Michelle Hilgart \\
\hline Chung-Yi Chiu & Matthew Hocking \\
\hline Aimee Christie & Megan Hosey \\
\hline Julie Chronister & Sigmund Hough \\
\hline Angela Ciccia & Matt Howard \\
\hline Brigit Clancy & Abbey Hughes \\
\hline \multicolumn{2}{|l|}{ Ram Cnaan } \\
\hline Wesley Cole & Todd Jackson \\
\hline Martha Combs & Alicia January \\
\hline John Connolly & Chad Johnson \\
\hline Andrew Cook & Brian Johnson \\
\hline Ashley Craig & Salene Jones \\
\hline Christopher Cranston & Shannon Juengst \\
\hline Melissa Day & Claire Kalpakjian \\
\hline Nicole Ditchman & Stuart Kamenetsky \\
\hline Bridget Dolan* & Robert Karol \\
\hline Ewa Domagała-Zyśk & Mary Kennedy \\
\hline Natalie Dong & Thomas Kerkhoff \\
\hline Diana Dorstyn & Lauren Khazem* \\
\hline Lucas Driskell & Elizabeth Kilgour \\
\hline Jennifer Duchnick & Sonya Kim \\
\hline Katherine Dunn* & Kristine Kingsley \\
\hline
\end{tabular}

John Kirk

Daniel Klyce

Nikos Konstantinou

A. Zarina Kraal

Megan Kramer

N. Erkut Kucukboyachi*

Simon Kunz*

Greg Lamberty

Eun-Jeong Lee

Injung Lee*

Margaret Legarreta

Kathleen Lemanek

Chao Li

Hanoch Livneh

Deirdre Logan

Eva Louvet

Emily Lund

Gloria $\mathrm{Ma}^{*}$

Victor Mark

Sonya Marshall-Gradisnik

Phillip Martin

Kathleen Martin Ginis

Gillian Mayersohn

John McClure

Scott McDonald

Susan McGurk

Ashlee McKeon

Joanna McParland

Michelle Meade

Swati Mehta

Elena Mendoza

Nancy Merbitz

Aaron Mertes*

Anne Michalek

Megan Miller

Michelle Mlinac*

Ivan Molton

Kimberley Monden

Helene Moriarty

Chelsea Morse

Stephanie Moser

Rachel Mueller

Gregory Murphy

Michelle Nario-Redmond

Sarah Nelson

Barry Nierenberg

Karen Nieves-Lugo

Anne Norup

Margaret Nosek
Nicholas Pastorek

Claudio Peter

Maurizio Pompili

Barnaby Proctor

Jennifer Reesman

Dominique Reinwand

J. Scott Richards

Elizabeth Richardson

Ann Marie Roepke*

Daniel Rohe

Shamira Rothmiller*

Cynthia Salorio

Jennifer Sánchez

Alecia Santuzzi

Jessica S. Schachter*

Michael Schüpbach

Ron Seel

Danielle Shapiro

Robert Shaw

Arielle Silverman

Richard Skolasky

Keith Slifer

Evan Smith*

Beth Springate

Lara Stepleman

William Stiers

David Strauser

Lauren Stutts

Stacy Suskauer

Shane Sweet

Alexandra Terrill

Marta Tremolada

Phil Ullrich

Anna Urbanowicz

Caroline Van Heugten

David Vance

Noa Vilchinsky

Jennifer Voth

Catherine Wilson

Winnie Wing-Sze Mak

Virgil Wittmer

Steven Woods

Anna Yam

Alan York

Amanda Young

\footnotetext{
* Denotes Co-reviewer
} 